The gradiometer of a SQUID-system for measuring very small biomagnetic signals should be made as insensitive as possible to the much larger uniform background fields. The balance of a gradiometer can be improved considerably by modifying the effective area of the gradiometer loops by a system of small adjustable superconducting plates.

This paper is intended as an aid for designing such a balancing system. We calculated the effective area of a gradiometer loop perpendicular to a rectangular or parallel to a circular plate. The paper contains graphs showing how large the plates have to be and where they must be placed.

The calculations show that for accurate balancing the distance of a plate to the wire of a loop must be large and that the plates must be as small as possible.

\title{
The design of a system of adjustable superconducting plates for balancing a gradiometer
}

\author{
J.A. Overweg and MI.J. Walter-Peters
}

In a SQUID-magnetometer system external magnetic fields are coupled into the SQUID by a fluxtransformer. ${ }^{1}$ If the magnetic fields to be measured are much weaker than other low gradient ambient fields, eg in our case of magnetocardiography in an unshielded environment, this fluxtransformer is usually a first or second order gradiometer ${ }^{2}$ as sketched in Fig. 1. When the gradiometer is ideal only inhomogeneous fields such as that of the human heart induce a current in the fluxtransformer and therefore give a flux in the SQUID. The total magnetic flux through all loops due to a homogeneous field should be zero, so that these fields are not measured.

However any real gradiometer will always be more or less sensitive to uniform fields too. This imbalance can be caused by imperfect alignment and non-identical areas of the pickup loops. Magnetic fields can be picked up by the wires that connect the loops. Even a perfectly balanced gradiometer will respond to a homogeneous field when this is distorted by a superconducting body or by eddy-currents in normal metals, for example of the insulation of the cryostat.

There are various ways to reduce the sensitivity of a gradiometer to uniform magnetic fields. One of these methods is to change the effective area of the gradiometer loops by small adjustable superconducting plates. ${ }^{3}$ This paper deals with the design of such a balancing system.

\section{Improving gradiometer balance with superconducting} plates

Because magnetic fields are expelled from superconducting bodies (the Meissner-effect) a superconducting plate will distort a magnetic field. There are two ways to use this effect for adjusting the effective area of a gradiometer loop:

The authors are at Twente University of Technology, Physics Department, PO Box 217, Enschede, the Netherlands. Received 23 May 1978.

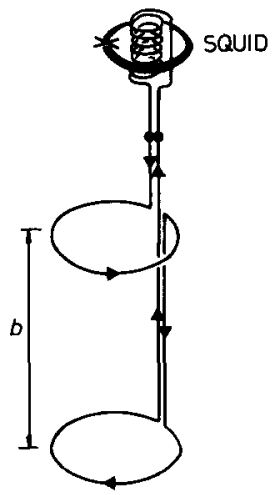

a

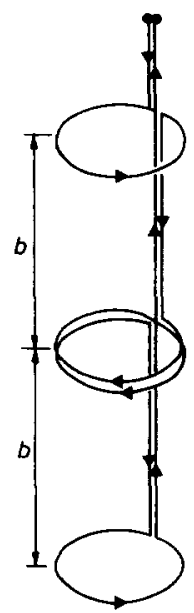

b

Fig. 1. a - first order gradiometer; $b$ - second order gradiometer

A plate parallel to the plane of the loop reduces its effective area to magnetic fields perpendicular to the loop (Fig. 2)

A superconducting plate perpendicular to the plane of the loop increases its effective area to fields parallel to the loop (Fig. 3).

The system of superconducting plates for balancing the gradiometer must be designed according to the following requirements. By properly adjusting the plates it must be possible to change the flux through the gradiometer loops in such a way, that the gradiometer becomes insensitive to uniform fields. The displacement of a plate that is required for a given flux-change through one of the loops must be as large as possible. This minimizes the effect of small vibrations of the plates and allows accurate balancing of the gradio- 


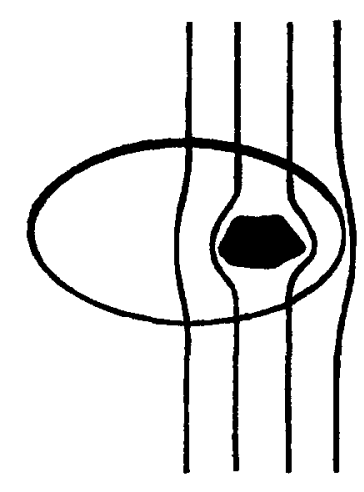

Fig. 2 Reduction of the effective area of a gradiometer loop to fields perpendicular to the loop by a superconducting plate

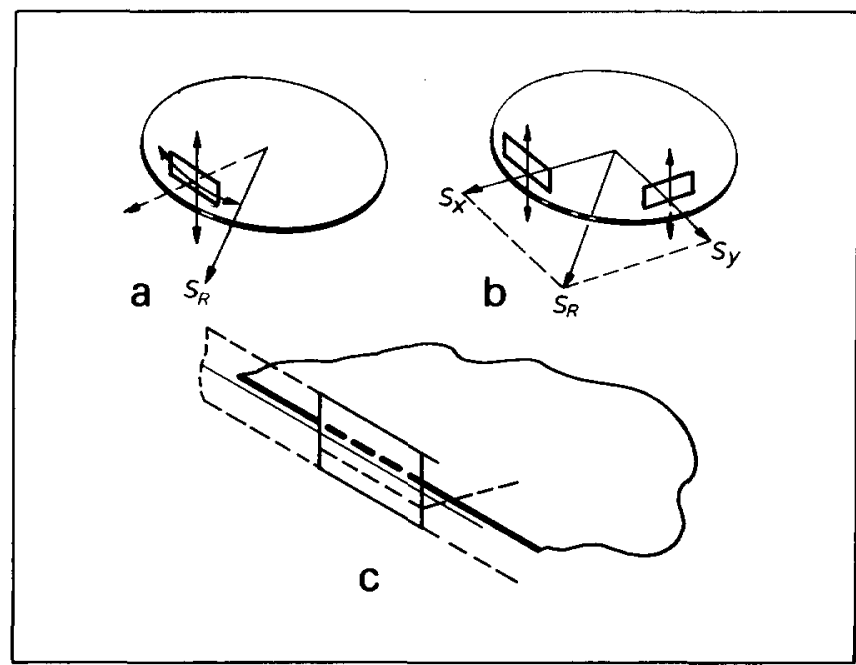

Fig. 3 Increasing the effective area of a loop to fields parallel to the loop by $a-$ one or $b-$ two rectangular superconducting plates, c - simplified two dimensional model of the loop and the plate

meter. It is desired that the system is constructed in such a way, that balancing the gradiometer in one direction changes the balance in perpendicular directions as little as possible.

Designing a system of small balancing plates according to these requirements implies answering the following questions:

how many plates are required?

what size and shape of plate should be chosen?

how should the plates relate to the gradiometer loops?

how far and in which direction have the plates to be moved in order to obtain the desired compensating effect?

In order to obtain an optimal configuration of balancing plates it will be necessary to calculate the change of the effective area of a circular loop of wire due to the presence of a superconducting plate as a function of its position and size. These calculations have to be made for different shapes of the plate and for various directions of the magnetic field. But some of these questions can be answered without extensive calculations. This is done in the next section.

\section{Number and shape of the plates}

The imbalance of a gradiometer can be represented by a vector $\bar{S}$, defined in such a way that the total magnetic flux through the gradiometer due to a uniform magnetic field $B$ is given by:

$$
\Phi=\bar{S} \cdot \bar{B}
$$

The gradiometer is insensitive to any uniform magnetic field perpendicular to the imbalance-vector $\bar{S}$ so that in principle it only has to be balanced in the direction of $\bar{S}$.

In practice it is more convenient to decompose $S$ into two or three orthogonal components and to use separate balancing plates for each component. It is obvious to choose one component along the axis of the gradiometer ( $Z$-coordinate) and to balance $S_{\mathrm{Z}}$ with a small plate parallel to one of the gradiometer loops (Fig. 2). It is to be expected that the influence of small vibrations of a plate will decrease on increasing its distance to the wire of the gradiometer. Therefore the best position for the plate for balancing $S_{Z}$ seems to be on the axis of the gradiometer. Because of the symmetry its most convenient shape is then a circular disc; this allows a rotation of the plate during the balancing procedure, another advantage is that its compensating effect can be calculated exactly.

The radial imbalance can be compensated by one or two rectangular plates perpendicular to one of the loops. One plate is sufficient if it can be moved independently in two directions: along the circumference of the gradiometer loop to place it perpendicular to $S_{\mathrm{R}}$ and parallel to itself to adjust its compensating effect (Fig. 3a). If two separate plates are used each plate needs to be adjustable in only one direction (Fig. 3b). Although there is a difference in balancing procedure both methods may give the same improvement in gradiometer balance.

More detailed calculations of the effect of the plates are now required. These calculations are presented in the next sections.

\section{Mathematical model of the balancing plates}

First some simplifying assumptions are necessary:

1. The plates are considered to be infinitely thin, so that only magnetic fields perpendicular to the plates are distorted.

2. A superconducting plate is represented by an ideally diamagnetic body $\left(\mu_{\mathrm{r}}=0\right)$. The magnetic field in the neighbourhood of a plate can then be calculated by solving Maxwell's equations.

3. We assume, that there are no free currents, that the fields change quasistatically and that outside the superconducting plates $\mu_{\mathrm{r}}=1$. Under these assumptions Maxwell's equations reduce to:

$\operatorname{div} \bar{B}=0$ and

$\operatorname{rot} \bar{B}=0$

These equations imply that $\bar{B}=-\operatorname{grad} U$, where $U$ is a scalar potential, that satisfies Laplace's equation:

$$
\nabla^{2} U=0
$$

As the magnetic field cannot penetrate the superconducting plate the normal component of $\bar{B}$ must vanish on its surface:

$$
B_{\mathrm{n}}=-\frac{\partial U}{\partial n}=0
$$


The boundary condition at infinity is, that the magnetic field is uniform. If for example the undistorted field is along the $x$-coordinate this is equivalent to:

$$
U(\infty)=-\mathrm{B}_{\infty} x
$$

The magnetic field-pattern for a given plate can be calculated if the corresponding boundary-value problem (2), (3), (4) is solved. Note that the velocity-potential that describes the distortion by some rigid obstacle of a uniform, incompressible and non-viscous fluid flow obeys exactly the same equations.

We now consider separately a gradiometer loop with a perpendicular rectangular plate and with a parallel circular plate. A uniform field $B_{\infty}$ is perpendicular to the plate and the magnetic flux through the loop has to be calculated as a function of the position and the size of the plate.

\section{The rectangular plate}

The plate is assumed to be small compared to a gradiometer loop. The part of the wire near the plate can then be represented by a straight line parallel to the plate, bounding a semi-infinite half plane (Fig. 3c). Furthermore we assume that the length of the rectangular plate is much larger than its height, so that end effects can be neglected. This makes the field pattern near the plate two-dimensional and the plate can be considered as a piece of an infinitely long strip.

Fig. 4 shows a cross-section of the plate and the idealized gradiometer loop. The height of the plate is $4 \mathrm{~b}$. The middle of the plate is situated at the origin of the coordinate system and the position of the wire of the loop is $\left(x_{1}, y_{1}\right)$.

The behaviour of the magnetic field near the plate can be derived from the rather simple field pattern of a long superconducting cylinder in a uniform field by a conformal transformation. ${ }^{4}$

The transformation yields the components of $\bar{B}$ along the coordinate axes of the plate $x$ and $y$ as a function of the old cylindrical coordinates $\rho$ and $\theta$.

$$
\begin{aligned}
& B_{\mathrm{x}}=\frac{B_{\infty}\left(\rho^{4}-b^{4}\right)}{\left(\rho^{2}-b^{2}\right)^{2}+4 b^{2} \rho^{2} \cos ^{2} \theta} \\
& B_{\mathrm{y}}=\frac{-2 B_{\infty} b^{2} \rho^{2} \sin 2 \theta}{\left(\rho^{2}-b^{2}\right)^{2}+4 b^{2} \rho^{2} \cos ^{2} \theta}
\end{aligned}
$$

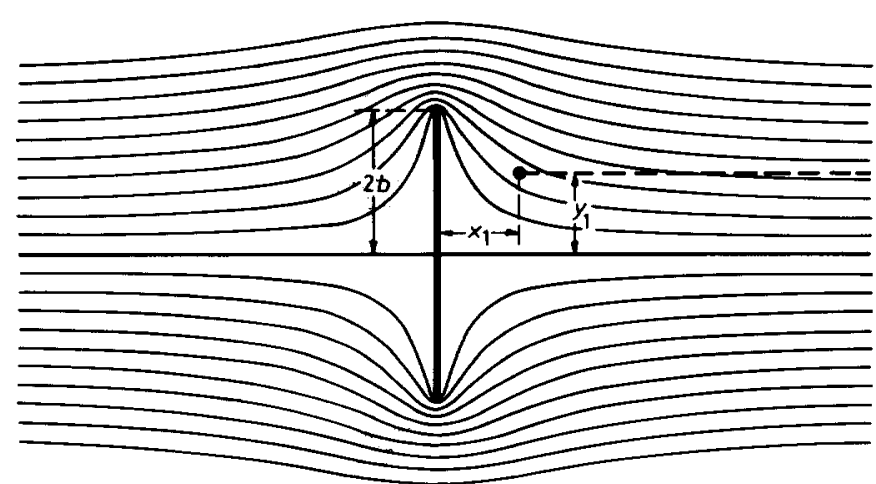

Fig. 4 Cross section of the plate of height $4 \mathrm{~b}$ and the idealized loop in a uniform magnetic field parallel to the loop
The coordinates $x$ and $y$ are related to $r$ and $\theta$ by:

$$
\begin{aligned}
& x=\left(\rho-\frac{b^{2}}{\rho}\right) \cos \theta \\
& y=\left(\rho+\frac{b^{2}}{\rho}\right) \sin \theta
\end{aligned}
$$

It is very complicated to express $B_{\mathbf{x}}$ and $B_{\mathbf{y}}$ explicitely as functions of $x$ and $y$, but this is not necessary, since we are only interested in the magnetic flux through the (idealized) gradiometer loop perpendicular to the plate (Fig. 4). For a plate of length $l$ this flux is given by:

$$
\begin{aligned}
\Phi_{\mathrm{y}}\left(x_{1}, y_{1}\right) & =l \int_{x=x_{1}}^{\infty} B_{\mathrm{y}}\left(x_{1}, y_{1}\right) \mathrm{d} x \\
& =l \int_{\rho=\rho_{1}}^{\infty} B_{\mathrm{y}}(\rho, \theta(\rho)) \frac{\mathrm{d} x}{\mathrm{~d} \rho} \mathrm{d} \rho
\end{aligned}
$$

Where $\rho_{1}$ is the solution of (7) and (8) for $x_{1}$ and $y_{1}$ and $\theta$ is related to $\rho$ by (8)

$$
\sin \theta=\frac{\rho y_{1}}{\rho^{2}+b^{2}}
$$

Differentiation to $\rho$ of (7) and (8) (with $y=y_{1}$ ) yields:

$$
\begin{aligned}
& \frac{\mathrm{d} x}{\mathrm{~d} \rho}=\frac{\rho^{2}+b^{2}}{\rho^{2}} \cos \theta-\frac{\rho^{2}-b^{2}}{\rho} \sin \theta \frac{\mathrm{d} \theta}{\mathrm{d} \rho} \\
& 0=\frac{\rho^{2}-b^{2}}{\rho^{2}} \sin \theta+\frac{\rho^{2}+b^{2}}{\rho} \cos \theta \frac{\mathrm{d} \theta}{\mathrm{d} \rho}
\end{aligned}
$$

After eliminating $\frac{\mathrm{d} \theta}{\mathrm{d} r}$ we obtain:

$$
\frac{\mathrm{d} x}{\mathrm{~d} \rho}=\frac{\rho^{2}+b^{2}}{\rho^{2}} \cos \theta+\frac{\left(\rho^{2}-b^{2}\right)^{2}}{\rho^{2}\left(\rho^{2}+b^{2}\right)} \frac{\sin ^{2} \theta}{\cos \theta}
$$

which can be rewritten as:

$$
\frac{\mathrm{d} x}{\mathrm{~d} \rho}=\frac{\left(\rho^{2}-b^{2}\right)^{2}+4 b^{2} \rho^{2} \cos ^{2} \theta}{\rho^{2}\left(\rho^{2}+b^{2}\right) \cos \theta}
$$

This is, together with (6) substituted into (9):

$$
\Phi_{y}\left(x_{1}, y_{1}\right)=l \int_{\rho_{1}}^{\infty} \frac{-4 B_{\infty} b^{2} \sin \theta}{\rho^{2}+b^{2}} \mathrm{~d} \rho
$$

With (10) the factor $\sin \theta$ is eliminated:

$$
\Phi_{y}\left(x_{1}, y_{1}\right)=-4 l B_{\infty} b^{2} y_{1} \int_{\rho_{1}}^{\infty} \frac{\rho}{\left(\rho^{2}+b^{2}\right)^{2}} \mathrm{~d} \rho
$$

From which follows:

$$
\Phi_{\mathrm{y}}\left(x_{1}, y_{1}\right)=\frac{-2 B_{\infty} b^{2} l y_{1}}{\rho_{1}^{2}+b^{2}}
$$




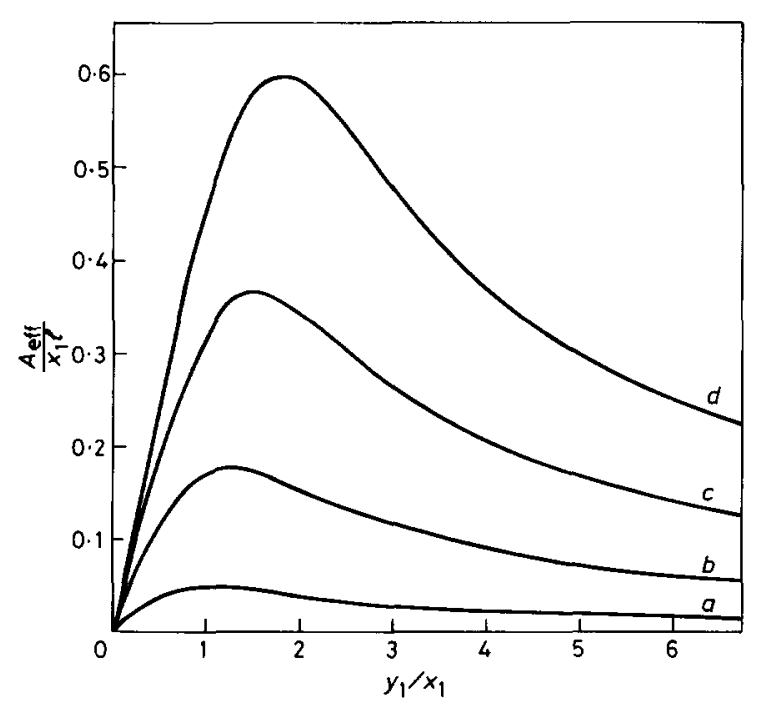

Fig. 5 Effective area of a loop with a rectangular plate, fields parallel to the loop as a function of the position of the plate for a few values of the height of the plate $4 b: a-b / x_{1}=0.22 ; b-0.43$ c $-0.65 ; \mathrm{d}-0.87$

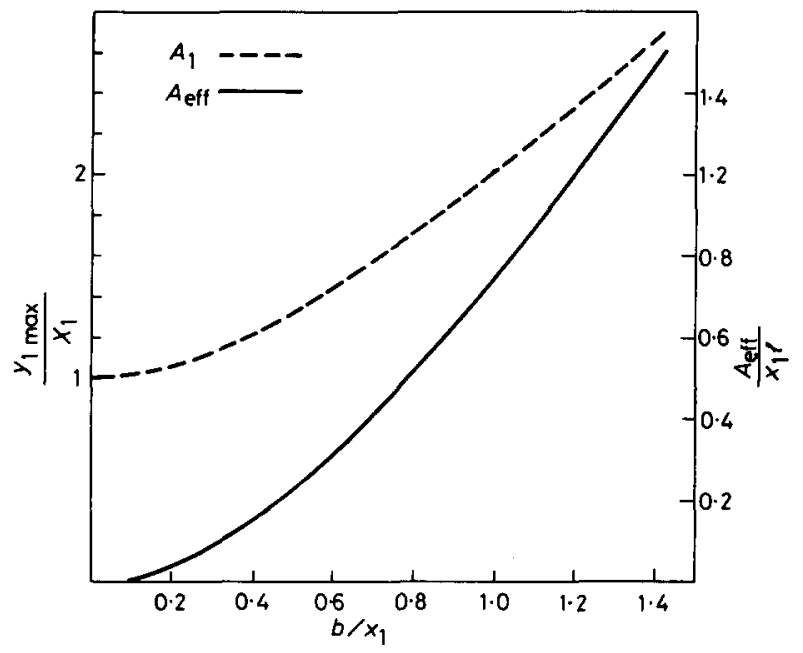

Fig. 6 Maximum effective area to fields parallel to the loop and the vertical position of the rectangular plate at which this maximum occurs as a function of the height of the plate $4 \mathrm{~b}$

Where $\rho_{1}$ is the solution of (7) and (8) for the given position $\left(x_{1}, y_{1}\right)$ of the loop relative to the plate.

The effect of the plate can also be represented by an effective area $A_{\text {eff }}$ defined as $\left|\Phi_{y}\left(x_{1}, y_{1}\right)\right| / B_{\infty}$ :

$$
A_{\text {eff }}\left(x_{1}, y_{1}\right)=\frac{2 b^{2} l y_{2}}{\rho_{1}^{2}+b^{2}}
$$

Fig. 5 shows the behaviour of $A_{\text {eff }}$ as a function of $y_{1}$ for a few values of $b$. Each value of $A$ eff between zero and a certain maximum can be obtained for two values of $y_{1}$. In a practical balancing system only the larger values should be used because then $\partial A_{\text {eff }} / \partial y$ is much smaller (requirement 2).

For these values of $y_{1}$ the effect of small variations in $x_{1}$ is also relatively small.

In Fig. 6 the maximum effective area which may be attained and the value of $y_{1}$ at which this maximum occurs are given as a function of plate dimensions.
The calculations have been checked experimentally and the measurements agree very well with the calculations. Due to the end effects the effective length of the plate is somewhat smaller than its real length, the difference being in the order of the height of the plate. If this effective length is substituted into (13) the calculations of $A_{\text {eff }}$ agree within $10 \%$ with measured data.

\section{The circular plate}

We considered a disc of radius, $a$, in the origin of the coordinate system and at a distance $z_{1}$ parallel to this disc, a circular loop of radius $r_{1}$. A uniform field $B_{\infty}$ is perpendicular to the disc along the $z$-axis (Fig. 7).

The derivation of the potential $U$ that describes the distortion of a uniform field by a superconducting disc of radius $a$ is rather complicated. This calculation is carried out by Kochin 5 and will not be presented in this paper. The result is:

$$
U\left(\nu_{1} z\right)=-B_{\infty} z\left\{1+\frac{2}{\pi}\left(\frac{1}{\nu}-\operatorname{arccot} \nu\right)\right\}
$$

where $\nu$ is the positive root of:

$$
\frac{r^{2}}{1+\nu^{2}}+{\frac{z^{2}}{\nu^{2}}}^{2}=a^{2}
$$

The component of the magnetic field along the $z$-axis is:

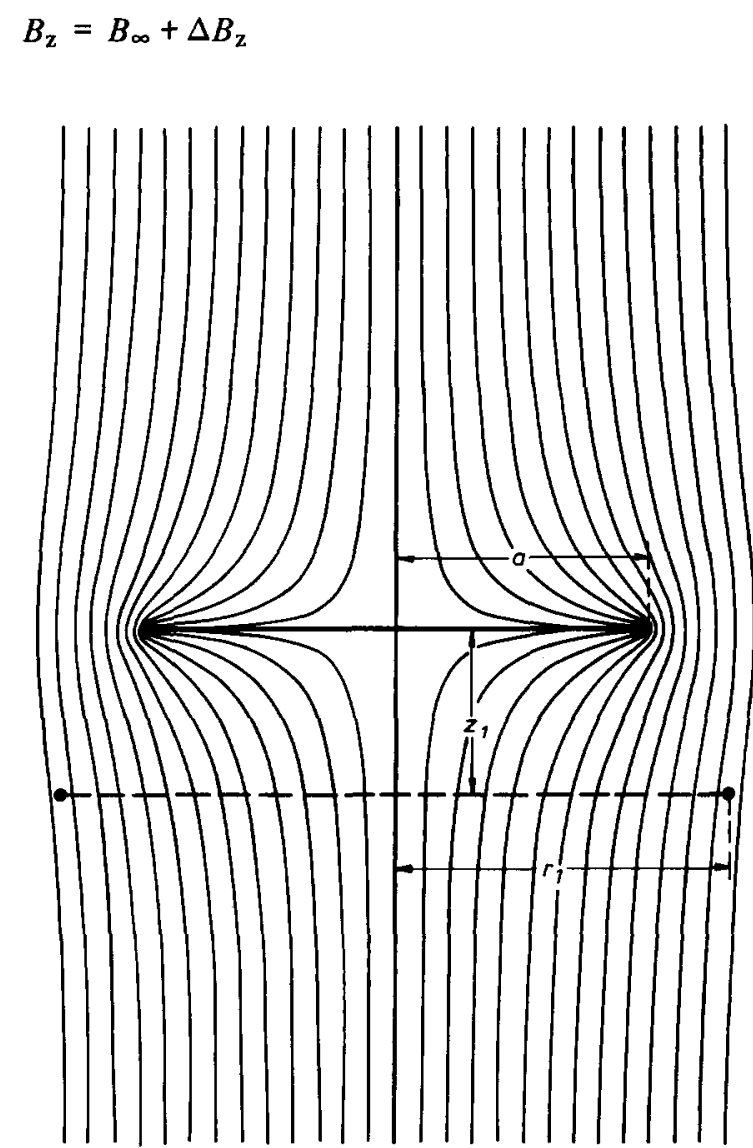

Fig. 7 Cross-section of the circular plate of radius a at a distance $z_{1}$ parallel to a loop of radius $r_{1}$ in a uniform magnetic field perpendicular to the loop 
where

$$
\begin{aligned}
\Delta B_{\mathrm{z}} & =\frac{2 B_{\infty}}{\pi} \frac{\partial}{\partial z} z\left(\frac{1}{\nu}-\operatorname{arccot} \nu\right) \\
& =\frac{2 B_{\infty}}{\pi}\left(\frac{1}{\nu}-\operatorname{arccot} \nu-\frac{z}{\nu^{2}\left(\nu^{2}+1\right)} \frac{\partial \nu}{\partial z}\right)
\end{aligned}
$$

The derivative $\partial \nu / \partial z$ is calculated by differentiating (15) with respect to $v$ at constant $r$ :

$$
\frac{\partial \nu}{\partial z}=\frac{1 / \partial z}{\partial \nu}=\frac{z \nu\left(1+\nu^{2}\right)^{2}}{z^{2}\left(1+\nu^{2}\right)^{2}+\nu^{4} r^{2}}
$$

This is substituted into (16)

$$
\begin{aligned}
\grave{\Delta B_{z}} & =\frac{2 B_{\infty}}{\pi} \frac{1}{\nu}-\left[\operatorname{arccot} \nu+\frac{z\left(1+\nu^{2}\right)}{z^{2} \nu\left(1+\nu^{2}\right)+\nu^{5} r^{2}}\right] \\
& =\frac{2 B_{\infty}}{\pi}\left[\frac{a^{2} \nu^{3}}{z^{2}+a^{2} \nu^{4}}-\operatorname{arccot} \nu\right]
\end{aligned}
$$

The magnetic flux through a loop of radius $r_{1}$ parallel to the disc is:

$$
\Phi_{\mathrm{Z}}=\Phi_{\infty}+\Delta \Phi_{\mathrm{z}}
$$

where $\Phi_{\infty}=\pi B_{\infty} r_{1}^{2}$

$$
\text { and } \begin{aligned}
\Delta \Phi_{\mathrm{z}} & =2 \pi \int_{r=0}^{r_{1}} r \Delta B_{\mathrm{z}}\left(r, z_{1}\right) \mathrm{d} r \\
& =2 \pi \int_{\nu_{0}}^{\nu_{1}} r(\nu) \Delta B_{\mathrm{z}}\left(\nu_{1} z_{1}\right) \frac{\mathrm{d} r}{\mathrm{~d} \nu} \cdot \mathrm{d} \nu .
\end{aligned}
$$

$v_{0}$ and $v_{1}$ are the solutions of (15) for $r=0$ and $r=r_{1}$. The derivative $\mathrm{d} r / \mathrm{d} \nu$ is calculated by differentiating (15) with respect to $\nu$ at constant $z$ :

$$
\frac{\partial r}{\partial \nu}=\frac{v r}{1+v^{2}}+\frac{z^{2}\left(1+v^{2}\right)}{r \nu^{3}}
$$

Now (17) and (19) are substituted into (18):

$$
\begin{aligned}
\Delta \Phi_{\mathrm{Z}}=4 B_{\infty} \int_{\nu_{0}}^{\nu_{1}} r^{2} & {\left[\frac{a^{2} \nu^{3}}{z^{2}+a^{2} \nu^{4}}-\operatorname{arccot} \nu\right] } \\
& {\left[\frac{\nu}{1+\nu^{2}}+\frac{z^{2}\left(1+\nu^{2}\right)}{r^{2} \nu^{3}}\right] \mathrm{d} \nu }
\end{aligned}
$$

With (15) $r^{2}$ is eliminated and after reduction we obtain:

$$
\begin{aligned}
\Delta \Phi_{\mathrm{Z}} & =4 B_{\infty} \int_{\nu_{0}}^{\nu_{1}}\left(\frac{a^{2} \nu^{3}}{z+a^{2} \nu^{4}}-\operatorname{arccot} \nu\right)\left(\frac{a^{2} \nu^{4}+z^{2}}{\nu^{3}}\right) \mathrm{d} \nu \\
& =4 B_{\infty} \int_{\nu_{0}}^{\nu_{1}}\left[a^{2}-a^{2} \nu \operatorname{arccot} \nu-\frac{z^{2}}{\nu^{3}} \operatorname{arccot} \nu\right] \mathrm{d} \nu
\end{aligned}
$$

This can readily be integrated to:

$$
\Delta \Phi_{Z}\left(\nu_{1}, z_{1}\right)=2 B_{\infty}\left[a^{2} \nu^{1}-\frac{z_{1}^{2}}{v_{1}}\right]\left[1-\left(\nu_{1}+\frac{1}{\nu_{1}}\right) \operatorname{arccot} \nu_{1}\right]
$$

(The primitive of (20) vanishes for $\nu_{0}=r^{2} / a^{2}-1$ )

With (15) this can be reduced to:

$$
\Delta \Phi_{Z}\left(r_{1}, z_{1}\right)=2 B_{\infty} r_{1}^{2}\left[\frac{\nu_{1}}{\nu_{1}^{2}+1}-\operatorname{arccot} \nu_{1}\right]
$$

where

$$
\begin{aligned}
v_{1}\left(r_{1}, z_{1}\right) & =\left\{\left[z_{1}^{2}+r_{1}^{2}-a^{2}+\left[\left(z_{1}^{2}+r_{1}^{2}-a^{2}\right)^{2}\right.\right.\right. \\
& \left.\left.\left.+4 a^{2} z_{1}^{2}\right]^{1 / 2}\right] / 2 a^{2}\right\}^{1 / 2}
\end{aligned}
$$

The effective area of the loop becomes:

$$
A_{\text {eff }}\left(r_{1}, z_{1}\right)=A_{0}+2 r_{1}^{2}\left(\frac{\nu_{1}}{\nu_{1}^{2}+1}-\operatorname{arccot} \nu_{1}\right)
$$

where $A_{0}=\pi r_{1}^{2}$ and $A_{\text {eff }}=A_{0}+\Delta A=A_{0}+\frac{\Delta \Phi_{\mathrm{z}}}{B_{\infty}}$

Fig. 8 shows $A_{\text {eff }} / A_{0}$ as a function of $z_{1} / r_{1}$ for two values of $a / \rho_{1}$. The graph shows that in order to minimize $\partial A_{\text {eff }} / \partial \mathrm{z}$ the disc should be made just large enough to attain the desired balancing effect. The maximum balancing effect is obtained for $z=0$ :

$$
\Delta A_{\max }=2 r_{1}^{2}\left[\frac{a\left(r_{1}^{2}-a^{2}\right)^{1 / 2}}{r_{1}^{2}}-\arcsin \frac{a}{r_{1}}\right]
$$

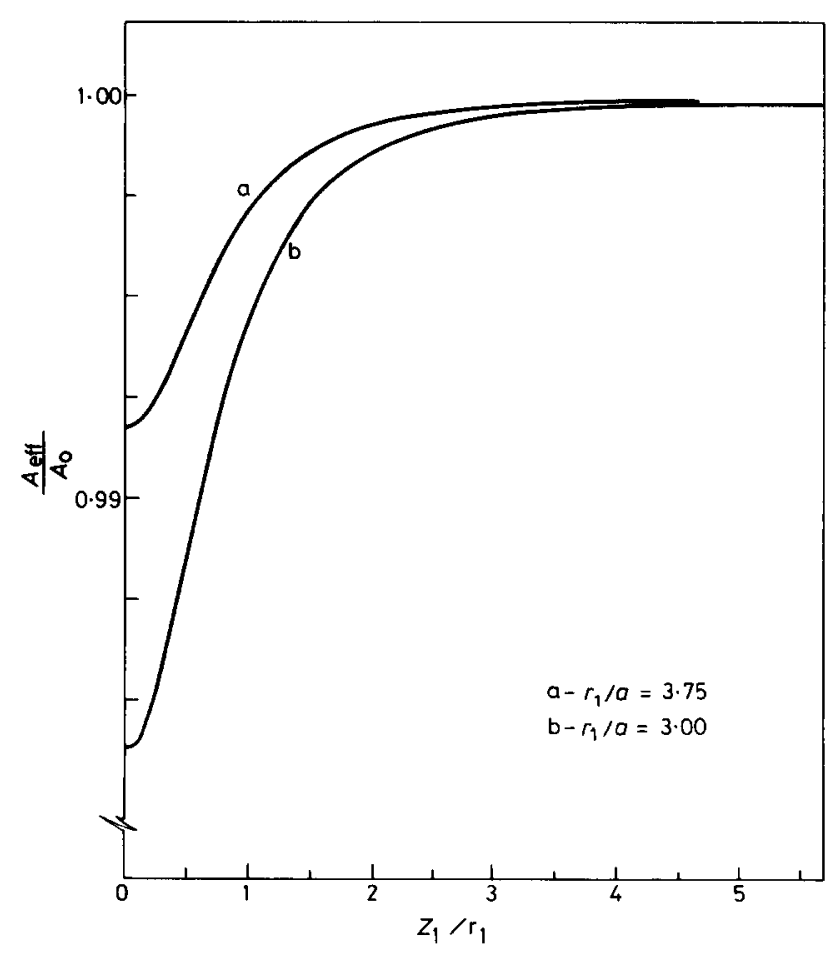

Fig. 8 Effective area of a loop with a parallel circular plate to a uniform field perpendicular to the loop as a function of the distance between the loop and the plate 


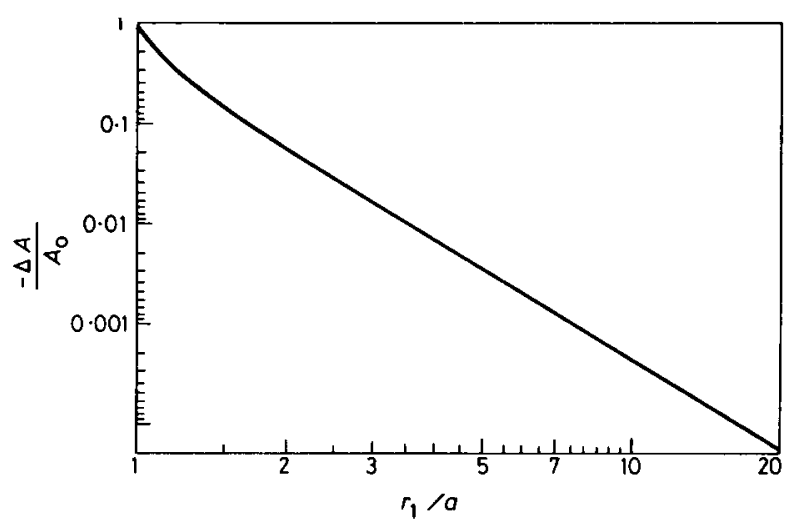

Fig. 9 Maximum change of the effective area of a circular loop due to a circular plate parallel to the loop as a function of the size of the plate

Fig. 9 shows $\Delta A_{\max }$ as a function of $r_{1} / a$.

These equations have also been checked experimentally and the agreement between calculations and measurements is even better than for the rectangular plate.

The experiments have been carried out with various 0.2 $0.4 \mathrm{~mm}$ thick Niobium and lead discs.

With a measuring accuracy of $5 \%$ no influence of the thickness or the material of the disc was observed. Even when the disc is larger than the loop, the measured data agree within measuring accuracy with the calculations.

\section{Conclusions}

It is possible to improve the balance of a nonideal gradiometer by at least two orders of magnitude with a system of two or three small adjustable superconducting plates.

The effect of such a plate can be calculated accurately and these calculations show, that the plates should be just large enough to obtain the desired balancing effect and that the rectangular plate should be at a large distance from the plane of the loop.

The authors are very grateful to Prof Dr L.C. van der Marel and Mrs Drs J.J. Wevers-Henke for their helpful discussions and suggestions.

\section{References}

1 Sarwinski, R.E. Cryogenics 17 (1977) 671

2 Opfer, J.E., Yeo, Y.K., Pierce, J.M., Rorden, L.H. IEEE Trans on Mag Mag-10 (1974) 599

3 Zimmerman, J.E., Frederick, N.V. NBS Report 10736 US Dept of Commerce (1972)

4 Eskinazi, S. Vector mechanics of fluids and magnetofluids J. Wiley, New York (1967)

5 Kochin, N.E., Roze, N.V., Kibel, I.A. Theoretical Hydrodynamics Interscience publ J Wiley, New York (1964) ch 7 